\title{
Development and evaluation of a loop-mediated isothermal amplification (LAMP) assay for quick identification of the Japanese salamander Hynobius tokyoensis
}

\author{
Tomoya Suzuki $^{1 *}$, Kanto Nishikawa $^{1,2}$, Yukuto Sato ${ }^{3,4,5}$ and Mamoru Toda ${ }^{4}$ \\ ${ }^{1}$ Graduate School of Global Environmental Studies, Kyoto University, Kyoto 606-8501, Japan \\ ${ }^{2}$ Graduate School of Human and Environmental Studies, Kyoto University, Kyoto 606-8501, Japan \\ ${ }^{3}$ Research Laboratory Center, Faculty of Medicine, University of the Ryukyus, Nishihara, \\ Okinawa 903-0125, Japan \\ ${ }^{4}$ Tropical Biosphere Research Center, University of the Ryukyus, Nishihara, Okinawa 903-0213, Japan \\ ${ }^{5}$ Center for Strategic Research Project, University of the Ryukyus, Nishihara, Okinawa 903-0213, Japan
}

(Received 24 June 2021, accepted 5 September 2021; J-STAGE Advance published date: 7 November 2021)

Species identification using molecular techniques has recently become common for various taxa. Loop-mediated isothermal amplification (LAMP) is one of the easiest and least expensive molecular identification methods. Although few studies have developed LAMP assays for amphibians, we believe that LAMP is also useful for identifying endangered amphibians. Hynobius tokyoensis and H. lichenatus occur in Honshu, Japan, and have parapatric distributions. They are similar morphologically, especially at early developmental stages, including eggs and larvae. Hynobius tokyoensis has been listed as a national endangered species in Japan since 2020, and unambiguous identification of these species is therefore important for their conservation and management. In this study, we developed a LAMP primer set for the mitochondrial cytochrome $\mathrm{b}$ region to detect $H$. tokyoensis, and we evaluated the LAMP assay using total genomic DNA from four $H$. tokyoensis and three $H$. lichenatus individuals from across most of their ranges. Our LAMP primer set could distinguish these two species. This study should help to establish LAMP assays for other endangered species and morphologically similar species.

Key words: Hynobius, LAMP, endangered species, cytochrome b, species identification

Accurate identification of species is necessary to understand and assess biodiversity. However, species identification often needs knowledge and skill. Some endangered species are morphologically similar to closely related species, and their identification is difficult (e.g., the Japanese giant salamander, Andrias japonicus, and Chinese giant salamander, A. davidianus; Fukumoto et

\footnotetext{
Edited by Masato Nikaido

* Corresponding author. suzuki.tomoya.5j@kyoto-u.ac.jp DOI: https://doi.org/10.1266/ggs.21-00046
}

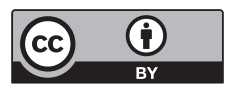

Copyright: (02021 The Author(s). This is an open access article distributed under the terms of the Creative Commons BY 4.0 International (Attribution) License (https://creativecommons.org/ licenses/by/4.0/legalcode), which permits the unrestricted distribution, reproduction and use of the article provided the original source and authors are credited. al., 2015). In these situations, accurate species identification is important for the conservation of endangered species (Yoshikawa et al., 2012). Species identification using molecular techniques has recently become common for various taxa (e.g., DNA barcoding; Ivanova et al., 2007; Smith et al., 2008; Che et al., 2012; Yano et al., 2020). While DNA sequencing is still expensive and requires special equipment, several alternative methods have been developed to facilitate genetic identification, such as polymerase chain reaction (PCR)-restriction fragment length polymorphism (Okamoto and Hikida, 2009; Igawa et al., 2015), PCR-amplified product length polymorphism (Shojo et al., 2015), and PCR-single-strand conformation polymorphism (Hayashi, 1992; Konstantinos et al., 2008).

Loop-mediated isothermal amplification (LAMP; Notomi et al., 2000) is one of the easiest molecular identification methods. Using a fluorescence detection 
reagent and blue light-emitting diode (LED)/ultraviolet (UV) rays, the operator can detect specific sequences by eye, obviating the need for special equipment to check the results. Furthermore, because the LAMP assay takes $60 \mathrm{~min}$ and its reaction temperature is $65{ }^{\circ} \mathrm{C}$, it can be done using a commercial electric kettle, instead of a thermal cycler. Therefore, the LAMP assay is a comparatively easy method for use not only by researchers but also by non-specialists. Because LAMP is quick and cheap, it has the potential to contribute to the conservation of endangered species, which requires the cooperation of professional citizens (e.g., police and government officials). LAMP assays have been developed for a few amphibians (Bufo japonicus japonicus, $B$. japonicus formosus and B. torrenticola; Eto et al., 2016), and we therefore believe that they could be useful for identifying endangered amphibians.

Hynobius tokyoensis is an endangered Japanese salamander endemic to the Kanto region of Honshu Island, Japan. Its population has decreased in recent decades because of urbanization (Sugawara et al.,2016). Hynobius lichenatus, which is closely related to $H$. tokyoensis, occurs in the Tohoku region of Honshu. These species are morphologically similar and they are distributed parapatrically in Honshu. At early developmental stages, they are difficult to distinguish morphologically.

In $2020, H$. tokyoensis was added to the list of national endangered species of Japan, and buying, selling or keeping it for commercial purposes is prohibited (Ministry of the Environment, Government of Japan, 2020; https:// www.env.go.jp/nature/kisho/domestic/list.html). In some cases, however, $H$. tokyoensis has been sold illegally as $H$. lichenatus, which it resembles morphologically. Therefore, accurate identification of $H$. tokyoensis is important for its conservation and management. Here, we developed a LAMP assay for the quick identification of H. tokyoensis. This was the first attempt to develop a LAMP primer set for a Hynobius salamander and the first attempt to assess the use of the LAMP assay to detect illegal trading of an endangered species.

We retrieved mitochondrial DNA (mtDNA) cytochrome b (cytb) sequences of $H$. tokyoensis $(\mathrm{n}=187)$ and $H$. lichenatus $(\mathrm{n}=5)$ from across almost the entire ranges of both species to cover the known intraspecific genetic variation from GenBank (AB266631-AB266661, AB266669-AB266671, JQ929926, LC004028-LC004101, and LC043222-LC043304; Matsui et al., 2007; Zheng et al., 2012; Sugawara et al., 2016). These sequences were aligned using ClustalW (Thompson et al., 1994) in MEGA ver. 7 (Kumar et al., 2016) and used to design primers. Comparing these sequences, we detected mutation sites by eye as candidate target regions. A LAMP primer set specific to the target region was designed with the online software PrimerExplorer V5 (http://primerexplorer. jp/lampv5e/; Table 1). A LAMP primer set needs six annealing positions (F1-3 and $\mathrm{B} 1-3)$; the inner primers (FIP/BIP) contain sequences of the sense (F2/B2) and antisense (F1/B1) strands of the target DNA (Table 1, Fig. 1). The outer primers are designed in regions F3 and B3. Therefore, the LAMP method needs four primers in total. Primers FIP and BIP, the loop structures, and positions $\mathrm{F} 1$ and $\mathrm{B} 1$ are important for the continuous amplification reactions of the LAMP method. Therefore, when we designed the primers, we selected annealing positions for primers $\mathrm{F} 1$ and $\mathrm{B} 1$ that contained comparatively many mutation sites (Fig. 1).

To validate the utility and accuracy of our LAMP primers, we used total genomic DNA from samples that were newly obtained for this study $(H$. tokyoensis from Metropolitan Tokyo, and Fukushima, Kanagawa and Chiba Prefectures; and H. lichenatus from Aomori, Fukushima and Gunma Prefectures). The LAMP reaction was carried out in a $25-\mu$ l reaction solution containing $1.0 \mu \mathrm{l}$ of template DNA, $4.6 \mu \mathrm{l}$ of distilled water (DW), $14.0 \mu \mathrm{l}$ of dNTPs $(2.5 \mathrm{mM}), 0.4 \mu \mathrm{l}$ each of primers FIP_cytb_Ht and BIP_cytb_Ht, $0.05 \mu \mathrm{l}$ each of primers F3_cytb_Ht and B3_cytb_Ht, $2.5 \mu \mathrm{l}$ of $10 \times$ Bst DNA Buffer, $1.0 \mu \mathrm{l}$ of Bst DNA Polymerase (Nippon Gene) and $1.0 \mu \mathrm{l}$ of Loopamp Fluorescent Detection Reagent (Eiken Chemical). The reaction mixtures were incubated at $65{ }^{\circ} \mathrm{C}$ for $60 \mathrm{~min}$ and then heated at $95{ }^{\circ} \mathrm{C}$ for 5 min to terminate the reaction. The reactions were performed on a MiniAmp thermal cycler (Applied Biosystems). After the reaction had finished, the fluorescence intensity of the reaction tube was checked under a commercial blue LED and under visible light. To evaluate the result for each tube, we compared the fluorescence intensity of the reaction tubes with that of a negative control (in which the template solution was replaced by DW) by eye.

We also conducted phylogenetic analyses based on the complete mtDNA cytb (1,141 bp) dataset of Matsui et al. (2007), who performed phylogenetic analyses using

Table 1. Primer set designated for LAMP assay of $H$. tokyoensis

\begin{tabular}{cl}
\hline \hline Primer code & \multicolumn{1}{c}{ Sequence (5'-3') } \\
\hline FIP_cytb_Ht & GAAAAGAAAATGGAACGCAAAAAATCTGTCCAATGAATTTGAGGTG \\
BIP_cytb_Ht & TCTAATCGCAGGGGCAAGCATCGGTTATTTGAGCCGGTTTC \\
F3_cytb_Ht & CTACATCGGAGACTCGCT \\
B3_cytb_Ht & TCTATATTAGAGTAAATTCCTGTGG \\
\hline
\end{tabular}




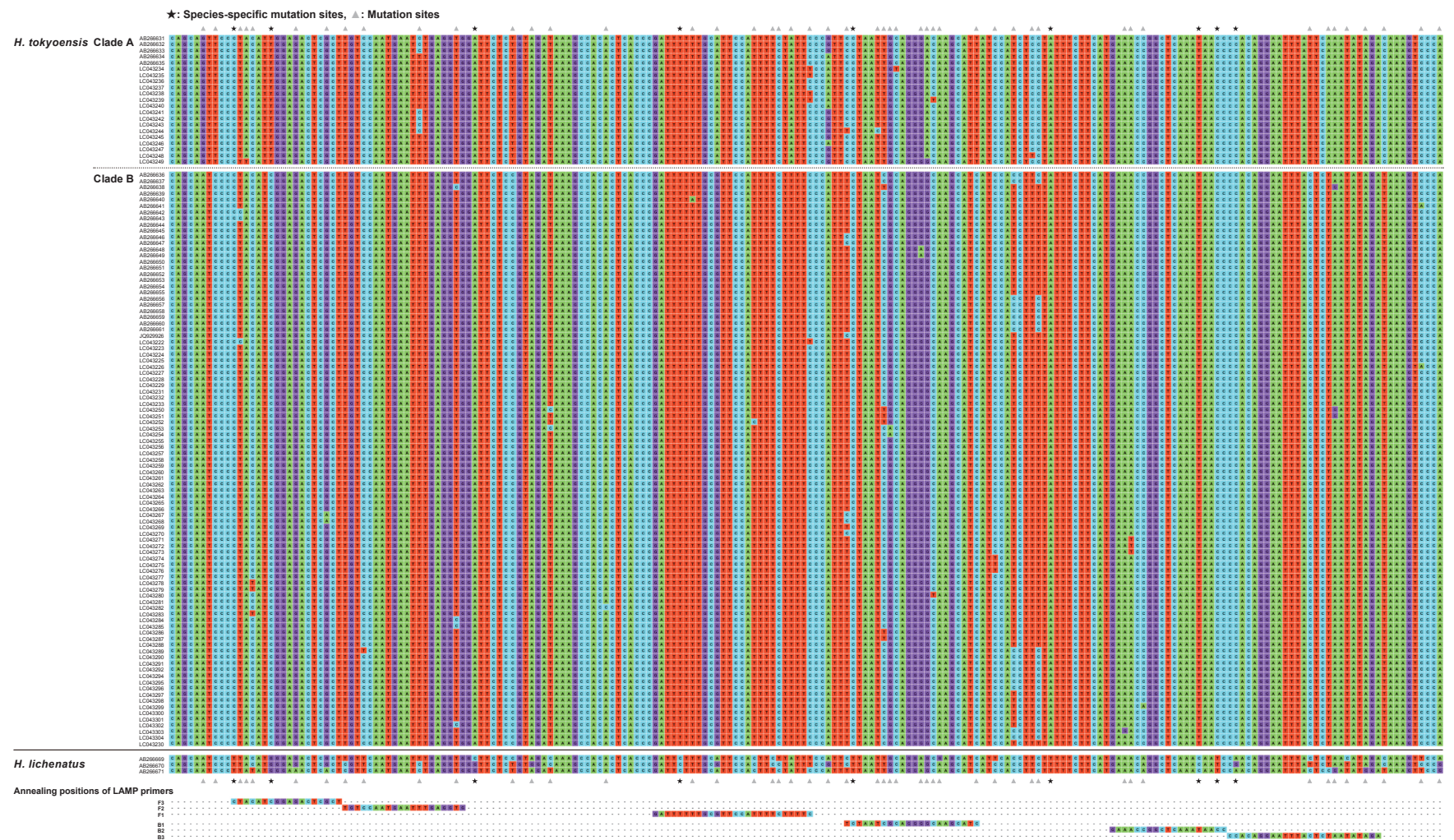

Fig. 1. Annealing positions of the H. tokyoensis LAMP primers and mtDNA cytb sequences of $H$. tokyoensis and H. lichenatus.

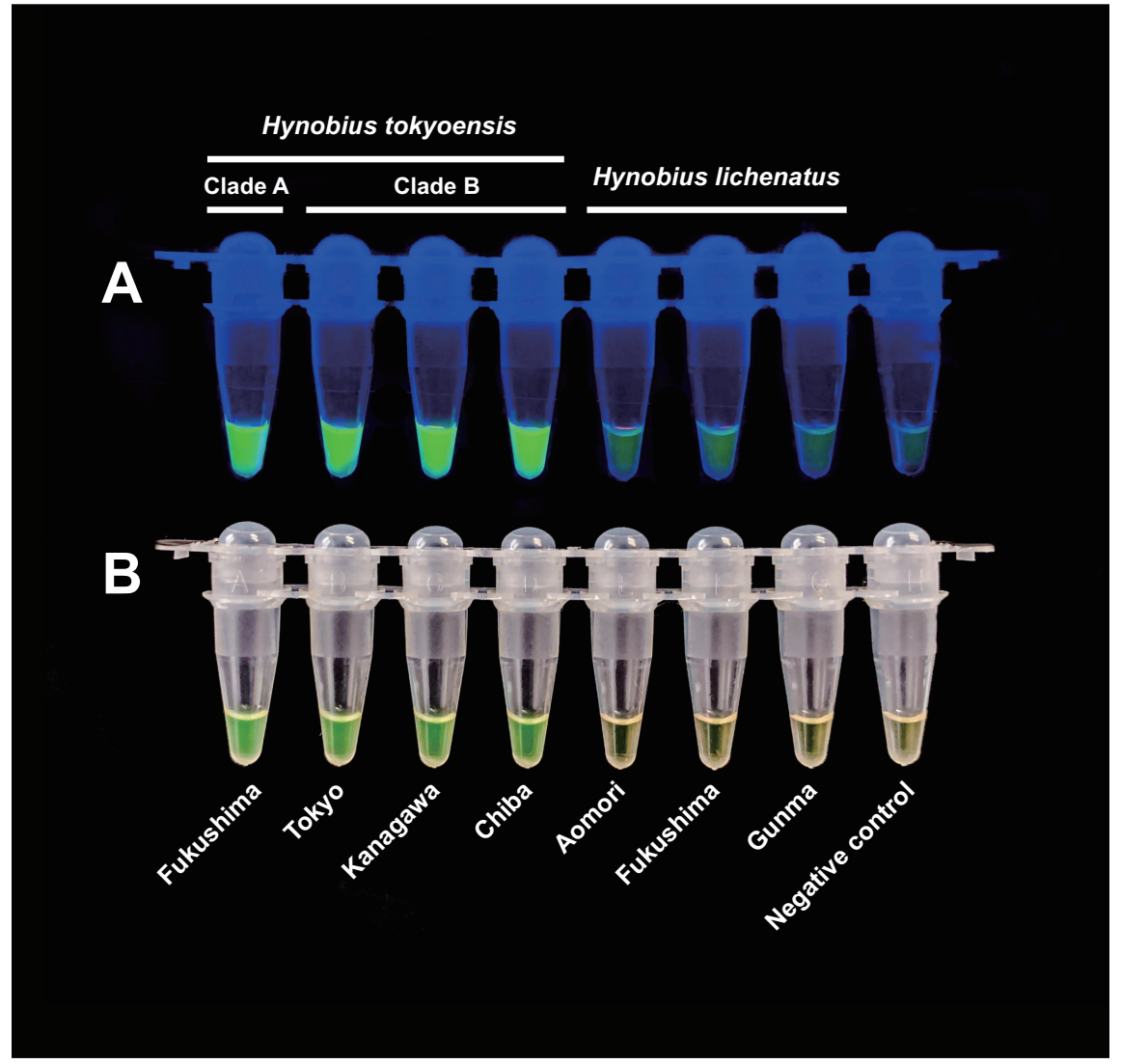

Fig. 2. Fluorescence intensity of the LAMP reactions. Photos of reaction tubes under blue LED (A) and visible (B) light. 
mtDNA $c y t b$ and control region sequences. However, we used only the mtDNA cytb dataset for the molecular phylogenetic analyses to confirm the phylogenetic relationship of $H$. tokyoensis and $H$. lichenatus based on the mtDNA cytb region, which was used to develop the LAMP primer set. Phylogenetic analyses were performed using the Bayesian (Huelsenbeck and Ronquist, 2001) method with MrBayes5D ver. 3.1.2 (Tanabe, 2008; http://www.fifthdimension.jp/) and the maximum likelihood (ML; Felsenstein, 1981) method using RAxML ver. 8.2.9 (Stamatakis, 2006). Nodal support was measured with the posterior probabilities in MrBayes5D, and 1,000 bootstrap replicates (Felsenstein, 1985) in RAxML. Before the ML and Bayesian phylogenetic estimations, we generated input files for RAxML and MrBayes5D using the program Kakusan4 (Tanabe, 2007; https://www.fifthdimension.jp/products/kakusan/) based on Schwarz's Bayesian Information Criterion (Schwarz, 1978) and the GTR + Gamma and HKY85 + Gamma models were selected, respectively. Bayesian analysis used 100 million Markov chain Monte Carlo cycles with a sampling frequency of 1,000 . Summary statistics for the estimated parameters were visualized and checked for convergence/stabilization using Tracer ver. 1.6 (Rambaut and Drummond, 2007; http://tree.bio.ed.ac.uk/software/ tracer/). To obtain a consensus tree, data from the first 10 million cycles were discarded as burn-in. As previous studies detected two genetically differentiated lineages in $H$. tokyoensis based on the mtDNA cytb region (Matsui et al., 2007; Zheng et al., 2012; Sugawara et al., 2016), we also calculated the $p$-distance between each clade/species using MEGA ver. 7 (Kumar et al., 2016).

Figure 2 shows the fluorescence intensity of LAMP assay samples, which clearly differed between $H$. tokyoensis and $H$. lichenatus under both blue LED light and visible light. In the future, we will use this assay in non-laboratory spaces, such as in the field or inspection rooms. This result provides important information for simplifying our assay. Because the police often intend to arrest suspected lawbreakers, quick species identification without expert knowledge of amphibians would be useful.

In addition, the $p$-distance based on the mtDNA $c y t b$ complete region $(1,141 \mathrm{bp})$ between $H$. tokyoensis and $H$. lichenatus was calculated as 12.9-13.0\% (Fig. 3). Fur-

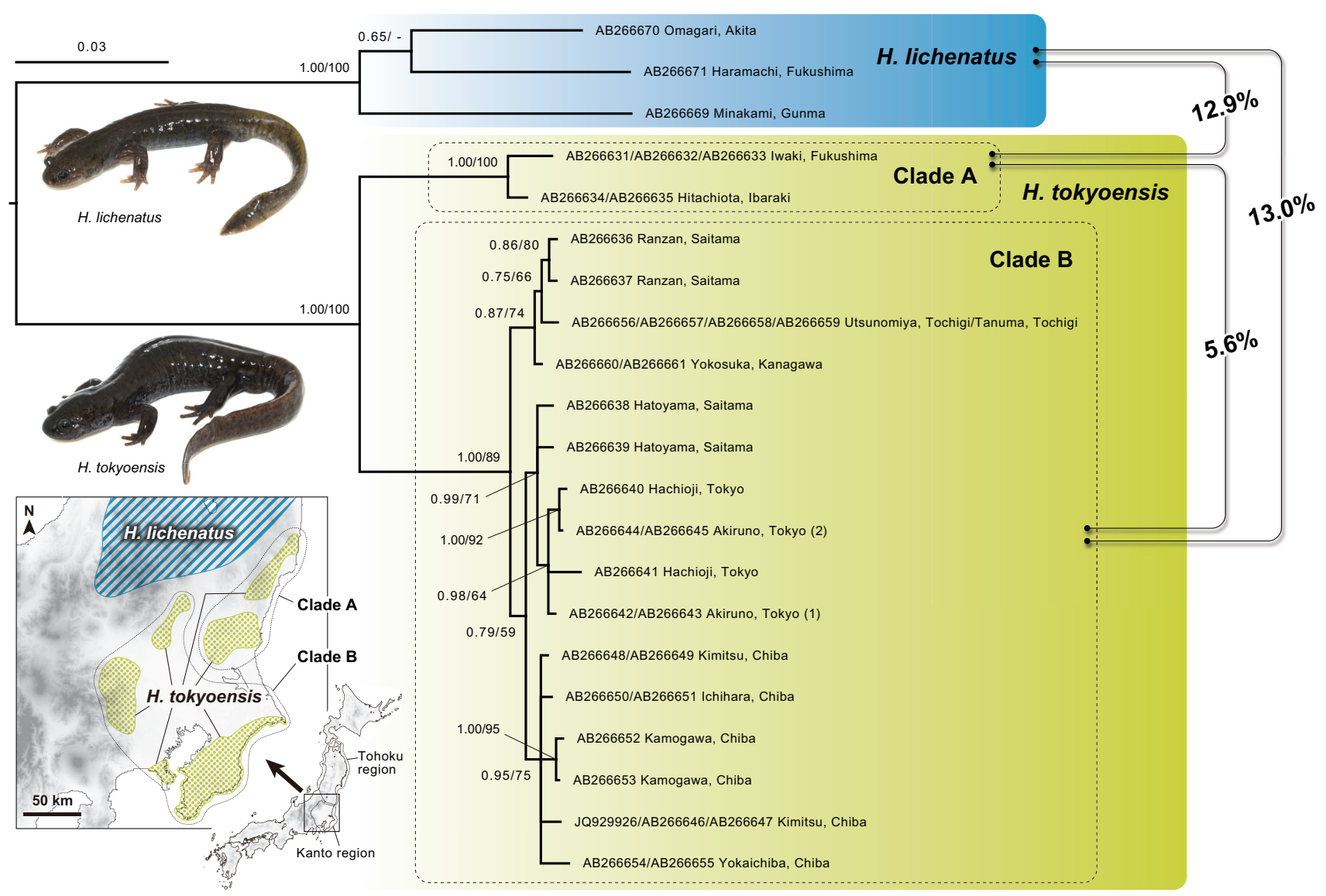

Fig. 3. Estimated Bayesian phylogenetic relationships of $H$. tokyoensis and H. lichenatus based on the mtDNA cytb region (1,141 bp) and a map of their distribution. The numbers at the nodes are the Bayesian posterior probabilities (left) and ML bootstrap support (right). The percentages between each clade are the averaged pairwise $p$-distance. 
thermore, the $p$-distance between two genetically distinct clades in $H$. tokyoensis that were detected in previous studies (Matsui et al., 2007; Zheng et al., 2012; Sugawara et al., 2016) was $5.6 \%$ (Fig. 3). We used samples from almost the entire range of both species to check the fluorescence intensity, and these samples corresponded geographically to members of all three genetic clades. Therefore, our LAMP primers for detecting $H$. tokyoensis can be used for both genetically differentiated clades within this species. Our results also imply that it is relatively easy to develop LAMP primer sets if the genetic differentiation between target species is $10 \%$ or more, but difficult if the genetic differentiation is $5 \%$ or less (Fig. 3). Because a LAMP primer set needs six annealing positions within 200-300 bp of the target DNA region (Notomi et al., 2000), the development of a species-specific LAMP primer set is comparatively difficult if the species are genetically closely related. Our new LAMP primer set included seven species-specific mutations. Furthermore, there were many mutations within the primer set that are not species-specific but were considered effective in the species-specific annealing reaction. We could find these annealing positions comparatively easily because the genetic distance between $H$. tokyoensis and $H$. lichenatus is large.

The identification of morphologically similar species using molecular approaches is important, and the LAMP assay is a quick and easy molecular identification method. Our LAMP primer set for H. tokyoensis should become very useful for identifying this species. The LAMP assay does not need special equipment such as a thermal cycler; a commercial electric kettle can be used, and an investigator can simply assess the DNA amplification results by eye. Due to its simplicity, some commercial detection kits have been developed (Mori and Notomi, 2009). The LAMP primer set developed here should be useful for detecting illegal trading of this species. If we develop a LAMP kit for practical $H$. tokyoensis identification, template DNA for a positive control must be included to assess experimental errors. We plan to consult government officials regarding practical application of this LAMP primer set. It may also be useful for detecting $H$. tokyoensis in a habitat using non-invasive sampling (e.g., using environmental DNA). We will evaluate whether our LAMP primer set can be used for detecting environmental DNA in a future study. This study is the first important step for conserving Japanese salamanders using a LAMP assay. We believe that molecular identification using LAMP assays can be adapted to many endangered species, and it will become a powerful, convenient tool for conserving endangered species.

We thank K. Takata, Y. Tomimori, K. Niwa and laboratory members for their help in collecting specimens. This research was supported by the Environment Research and Technology
Development Fund (JPMEERF20204002) of the Environmental Restoration and Conservation Agency of Japan.

\section{REFERENCES}

Che, J., Chen, H.-M., Yang, J.-X., Jin, J.-Q., Jiang, K., Yuan, Z.-Y., Murphy, R. W., and Zhang, Y.-P. (2012) Universal COI primers for DNA barcoding amphibians. Mol. Ecol. Res. 12, 247-258.

Eto, K., Matsui, M., Nishikawa, K., and Haramura, T. (2016) Development and evaluation of loop-mediated isothermal amplification (LAMP) assay for quick identification of three Japanese toads. Curr. Herpetol. 35, 33-37.

Felsenstein, J. (1981) Evolutionary trees from DNA sequences: a maximum likelihood approach. J. Mol. Evol. 17, 368-376.

Felsenstein, J. (1985) Confidence limits on phylogenies: an approach using the bootstrap. Evolution 39, 783-791.

Fukumoto, S., Ushimaru, A., and Minamoto, T. (2015) A basinscale application of environmental DNA assessment for rare endemic species and closely related exotic species in rivers: a case study of giant salamanders in Japan. J. Appl. Ecol. 52, 358-365.

Hayashi, K. (1992) PCR-SSCP: a method for detection of mutations. Genet. Anal. Tech. Appl. 9, 73-79.

Huelsenbeck, J. P., and Ronquist, F. (2001) MrBayes: Bayesian inference of phylogenetic trees. Bioinformatics 17, 754755 .

Igawa, T., Komaki, S., Takahara, T., and Sumida, M. (2015) Development and validation of PCR-RFLP assay to identify three Japanese brown frogs of the true frog genus Rana. Curr. Herpetol. 34, 89-94.

Ivanova, N. V., Zemlak, T. S., Hanner, R. H., and Hebert, P. D. (2007) Universal primer cocktails for fish DNA barcoding. Mol. Ecol. Notes 7, 544-548.

Konstantinos, K. V., Panagiotis, P., Antonios, V. T., Agelos, P., and Argiris, N. V. (2008) PCR-SSCP: a method for the molecular analysis of genetic diseases. Mol. Biotechnol. 38, 155-163.

Kumar, S., Stecher, G., and Tamura, K. (2016) MEGA7: Molecular evolutionary genetics analysis version 7.0 for bigger datasets. Mol. Biol. Evol. 33, 1870-1874.

Matsui, M., Tominaga, A., Hayashi, T., Misawa, Y., and Tanabe, S. (2007) Phylogenetic relationships and phylogeography of Hynobius tokyoensis (Amphibia: Caudata) using complete sequences of cytochrome $b$ and control region genes of mitochondrial DNA. Mol. Phylogenet. Evol. 44, 204-216.

Mori, Y., and Notomi, T. (2009) Loop-mediated isothermal amplification (LAMP): a rapid, accurate, and cost-effective diagnostic method for infectious diseases. J. Infect. Chemother. $\mathbf{1 5}, 62-69$.

Notomi, T., Okayama, H., Masubuchi, H., Yonekawa, T., Watanabe, K., Amino, N., and Hase, T. (2000) Loop-mediated isothermal amplification of DNA. Nucleic Acids Res. 28, e63.

Okamoto, T., and Hikida, T. (2009) Three genetic lineages of the Japanese skink Plestiodon japonicus (Scincidae, Squamata) and the genetic composition of their contact zones. J. Zool. Syst. Evol. Res. 47, 181-188.

Schwarz, G. (1978) Estimating the dimension of a model. Ann. Stat. 6, 461-464.

Shojo, H., Tanaka, M., Takahashi, R., Kakuda, T., and Adachi, N. (2015) A unique primer with an inosine chain at the 5 -terminus improves the reliability of SNP analysis using the PCR-amplified product length polymorphism method. PLoS One 10, e0136995.

Smith, M. A., Poyarkov, N. A., Jr., and Hebert, P. D. (2008) DNA barcoding: CO1 DNA barcoding amphibians: take the 
chance, meet the challenge. Mol. Ecol. Resour. 8, 235-246.

Stamatakis, A. (2006) RAxML-VI-HPC: maximum likelihoodbased phylogenetic analyses with thousands of taxa and mixed models. Bioinformatics 22, 2688-2690.

Sugawara, H., Kusano, T., and Hayashi, F. (2016) Fine-scale genetic differentiation in a salamander Hynobius tokyoensis living in fragmented urban habitats in and around Tokyo, Japan. Zoolog. Sci. 33, 476-484.

Tanabe, A. S. (2007) Kakusan: a computer program to automate the selection of a nucleotide substitution model and the configuration of a mixed model on multilocus data. Mol. Ecol. Notes 7, 962-964.

Thompson, J. D., Higgins, D. G., and Gibson, T. J. (1994) CLUSTAL W: improving the sensitivity of progressive multiple sequence alignment through sequence weighting, position-specific gap penalties and weight matrix choice. Nucleic Acids Res. 22, 4673-4680.

Yano, K., Takenaka, M., Mitamura, T., and Tojo, K. (2020) Identifying a "pseudogene" for the mitochondrial DNA COI region of the corixid aquatic insect, Hesperocorixa distanti (Heteroptera, Corixidae). Limnology 21, 319-325.

Yoshikawa, N., Matsui, M., Hayano, A., and Inoue-Murayama, M. (2012) Development of microsatellite markers for the Japanese giant salamander (Andrias japonicus) through next-generation sequencing, and cross-amplification in its congener. Conserv. Genet. Resour. 4, 971-974.

Zheng, Y., Peng, R., Murphy, R. W., Kuro-o, M., Hu, L., and Zeng, X. (2012) Matrilineal genealogy of Hynobius (Caudata: Hynobiidae) and a temporal perspective on varying levels of diversity among lineages of salamanders on the Japanese Islands. Asian Herpetol. Res. 3, 288-302. 